\title{
Residuos sólidos reciclables y reutilizables producido en la UNAH-CU y sus potenciales de uso
}

\author{
René Martínez Wong \\ Jorge Fernando Pacheco Dubón ${ }^{2}$ \\ Juan Carlos Montalván Flores ${ }^{3}$
}

\section{RESUMEN}

El objetivo principal del estudio fue determinar la cantidad de residuos sólidos reciclables y reutilizables que produce la UNAH-CU, en un periodo académico y sus potenciales de uso. Para ello se realizó un diseño de investigación mixto, con un instrumento de recolección cuantitativa que permitió obtener una cifra del total de residuos sólidos que produce la UNAH-CU por periodo académico, y un instrumento cualitativo que permitió vislumbrar las principales deficiencias y fortalezas en la construcción de un Sistema Integral de Gestión de Residuos Sólidos, para la clasificación, reciclaje y reutilización de los residuos, de tal modo potencializar iniciativas lúdicas que podrán contribuir a la del medio ambiental ambiente y la sociedad. Se encontró que la UNAH-CU posee una gran cantidad de recursos reutilizables que pueden servir para iniciativas lúdicas para próximos proyectos de investigación, vinculación y desarrollo medioambiental.

Palabras clave: residuos sólidos reciclables y reutilizables, gestión de residuos sólidos, hábitos medioambientales.

\footnotetext{
${ }^{1}$ Beneficiario de una beca básica de la DICYP, profesor de la Escuela de Arquitectura, Facultad de Humanidades y Artes, UNAH; rmwong54@hotmail.com

${ }^{2}$ Egresado de la Escuela de Sociología, Facultad de Ciencias Sociales, UNAH

${ }_{3}^{3}$ Profesional de la arquitectura, Cruz Roja Hondureña: jcmf87@gmail.com
} 


\section{ABSTRACT}

The main objective of the study was to determine the Amount of Recyclable and Reusable Solid Waste Produced by UNAH-CU in an Academic Period and Its Potential Use. For this, a mixed research design was carried out, with a quantitative collection instrument that allowed to obtain a figure of the total solid waste produced by UNAH CU for academic period, and a qualitative instrument that allowed to glimpse the main deficiencies and strengths in the construction Of an Integral System of Management of Solid Waste, for the classification, recycling and reuse of the residues, in order to potentiate ludic initiatives that can contribute to the environment and society. It was found that UNAH CU has many reusable resources that can be used for upcoming research, linkage and environmental development projects.

Keywords: recyclable and reusable solid waste, solid waste management, environmental habits. 


\section{INTRODUCCIÓN}

El presente artículo reúne los principales resultados de la investigación "Residuos sólidos reciclables y reutilizables producidos en la UNAH-CU por periodo académico y sus potenciales de uso" cuya necesidad responde a que en vista, que la UNAH cuenta con un cuerpo administrativo, docente y estudiantil, que suman a más de 50 mil personas, es necesario brindar atención especial a la cantidad de residuos que se generan y en la responsabilidad que como institución, como centro de enseñanza e innovación científica y como comunidad debe de atender bajo criterios de conciencia ambiental y responsabilidad ambiental.

Por lo cual, el presente artículo expone los datos recolectados a través del proceso de investigación, y la construcción de una base de datos sobre la cantidad total de residuos sólidos reciclables y reutilizables que produce la UNAH-CU por periodo académico, así como algunos potenciales para su uso

\section{METODOLOGÍA}

El alcance de la investigación es de tipo explicativo descriptivo, el tipo de investigación es de tipo cuantitativo con un componente cualitativo, lo que la convierte en una investigación de carácter mixto, El campo de estudio para la investigación fue desarrollado en UNAH-CU, realizando levantamientos cuantitativos a todos los edificios del campus ya sea administrativos y de docencia. Por esta razón se realizó un bosquejo por zonas para realizar la cobertura del campus de manera sistematizada (ver imagen 1) así medir la cantidad de residuos que produce la UNAH-CU en un periodo académico, la población que fue seleccionada para la obtención de los datos del levantamiento cualitativo, se levantó una serie de entrevistas al personal administrativo de mantenimiento y limpieza, ya que son los actores que intervienen en mayor medida en el manejo de los residuos; se clasificaron los residuos sólidos en 4 categorías, que constituyen las 4 variables principales del estudio: reutilizables, reciclables, orgánicos y no reutilizables ni reciclables. Posteriormente, se complementaron los datos cuantitativos con el análisis de los discursos (nivel cualitativo) de las personas involucradas en la gestión de los residuos en el campus, con el objetivo de verificar empíricamente las características, limitaciones y los marcos normativos del proceso de gestión de los residuos sólidos. Para el levantamiento de datos o la cuantificación de los residuos se utilizó una guía de observación como instrumento de recolección de información primaria, se utilizó Microsoft Excel para desarrollar las tablas y gráfi- 
cos en base a los datos obtenidos en la guía de observación, se contabilizaron (con libras y medida de peso global toneladas) y se caracterizaron los residuos producidos en cada una de las zonas y los edificios de la Ciudad Universitaria.

\section{RESULTADOS}

Partimos del supuesto de que no es congruente señalar propuestas o alternativas de reutilización y reciclaje de los residuos sólidos que produce la UNAH-CU, sin señalar conjuntamente las principales limitaciones que existen en el proceso de Gestión de Residuos Sólidos al interior de la Ciudad Universitaria. Por lo cual, la dimensión mixta de la investigación abrió la posibilidad de dar propuestas de uso a la cantidad de residuos producidos en la UNAH en un periodo académico, y al mismo tiempo, dar o proponer alternativas de mejoras para el proceso de gestión y clasificación de los residuos sólidos.

En la parte cualitativa de la investigación, se hizo un acercamiento empírico, a través de entrevistas semi-estructuradas aplicadas a actores claves, y el análisis de discurso como método de análisis, lo cual arrojo importante información sobre las principales limitaciones que existen en la implementación y puesta en marcha de un Sistema Integral de Residuos Sólidos, y en particular, en la dimensión de la clasificación de los residuos según su origen.

La información tiene su fundamento de validez en los años de experiencia de las y los principales actores que conforman la estructura actual de gestión de residuos. Los resultados de los datos recogidos se presentarán a continuación en 3 niveles, los cuales son: nivel de depósito, recolección y en el traslado.

En el nivel de depósito se encontró que existe una estructura física significativa destinada a servir como base para impulsar prácticas de clasificación de los residuos sólidos en el área del campus en Ciudad Universitaria. La estructura se vio reforzada en el año 2014 en el marco del programa "Universidad Verde", se instalaron alrededor de 34 contenedores pintados de los colores azul, verde y amarillo, que representan los principales niveles de tipificación de residuos (Orgánico, plástico, papel, vidrio y aluminio) y actualmente constituye el principal avance en materia de clasificación y reciclaje en la UNAH. Los contenedores se ubicaron en los edificios y puntos claves al interior de la UNAH para promover las prácticas de clasificación y lograr un cambio cultural en el hábito del depósito de los residuos, con cierta conciencia ambiental y 
cierto tipo de racionalidad ambiental más responsable, sin embargo, el proyecto no tuvo el impacto esperado.

Actualmente los residuos sólidos en el nivel de depósito no se clasifican debido en principio a las razones que se consideran a continuación:

La comunidad universitaria (particularmente los estudiantes) no utilizan los basureros de colores según su función, tal como lo expresa las personas que fueron entrevistadas, a continuación, resumo algunas expresiones:

Los estudiantes y los empleados, no respetan o no tienen la cultura de clasificar los residuos sólidos, ellos botan la basura en cualquier basurero.

La comunidad universitaria, tiene la costumbre de tirar la basura en cualquier lugar, incluyendo dentro de las aulas, teniendo que estar recolectando los desechos en bolsas comunes.

Esto representa un evidente problema de hábitos y un problema de sensibilización en la comunidad estudiantil universitaria sobre la importancia de depositar la basura en su lugar, contribuyendo directamente a la clasificación de la misma. Por lo que se necesita realizar un trabajo a nivel cultural, que logre fomentar una conciencia de reciclaje ligada a una racionalidad ambiental con mayor responsabilidad individual y colectiva sobre el impacto en el medio ambiente y las alternativas sobre el uso y tratamiento de los residuos sólidos, contribuyendo directamente al desarrollo sostenible y a la reducción del daño medioambiental.

Existe otro problema en el nivel de depósito relacionado directamente con la estructura del Sistema de Gestión de Residuos Sólidos. El procedimiento de depósito de los residuos, en un primer nivel, culmina cuando se depositan las bolsas que contienen los residuos en los contenedores más grandes, mismos que son recolectados posteriormente por el personal que opera los automóviles e insumos de recolección. Ocurre aquí, que los residuos se revuelven o mezclan indistintamente de su origen 0 tipificación. Por lo cual, si se realizara la clasificación de los residuos de parte del estudiantado y personal de aseo resultaría una tarea en vano sin ningún impacto real, por cual, para asegurar un proceso de clasificación se debe de tomar acciones de clasificación con todos los involucrados en todos los procesos del nivel de depósito. Por último, la estructura de gestión de residuos no cuenta con un centro de acopio en donde se depositen y almacenen los residuos clasificados. Por lo que un proceso de clasificación real en todo el nivel de depósito del proceso de gestión de residuos resulta imposible con la estructura actual con que cuenta la UNAH. La UNAH-CU no 
cuenta con equipo adecuado, ni un centro de acopio de los residuos sólidos y mucho menos como clasificarlos.

Un sistema de gestión de residuos exigiría concordancia en los diferentes niveles y procesos de gestión de los residuos. En el nivel de depósito y recolección se debe de asegurar la clasificación de los residuos para su posterior tratamiento, esto a través de las condiciones materiales necesarias o la estructura física.

Por último, cabe señalar que la principal tarea del personal de aseo con relación a los residuos es recolectarlos de los depósitos en los edificios y llevarlos a los contenedores mayores, donde el tren de aseo los recoge para luego trasladarlos al vertedero municipal.

En el nivel del traslado se encontró que la responsabilidad ambiental de la UNAH es mínima en relación a la gran cantidad de residuos que se producen al interior del campus, es decir, la universidad produce alrededor de 12 toneladas diarias de residuos sólidos de todo tipo, sin medir las consecuencias directas e indirectas que esto acarrea tanto para el medio ambiente como para la sociedad.

El traslado de los residuos constituye la fase final del proceso de gestión de residuos actual en la universidad, una vez concluido el proceso de recolección los residuos se trasladan al exterior de la UNAH, es decir al crematorio municipal y en ese lugar tampoco existe lugares de acopio según su clasificación, razón por la cual de nada sirve que se clasifique la basura, si en el crematorio se deposita en un solo lugar.

En el crematorio ocurren dinámicas de notable interés para la UNAH, el hecho de que los botes plásticos y aluminios que salen de la UNAH vayan a manos de miembros de maras y pandillas constituye una preocupación alarmante, ya que la institución está contribuyendo indirectamente al enriquecimiento y financiamiento de estos grupos criminales, el peso de la responsabilidad social y ambiental es innegable, por lo que la Universidad debe de velar por dar solución a este tipo de situaciones que contribuyen al crimen organizado y otras situaciones en el país. El principal problema en el nivel de traslado de los residuos refleja la condensación de las falencias y deficiencias del Sistema de Gestión de Residuos en sus dimensiones simbólicas y materiales en los dos primeros niveles, es decir, las prácticas y hábitos ambientales inadecuados y la estructura física insuficiente para la clasificación de los residuos define el destino final de los residuos, lo que ocasiona el desaprovechamiento de recursos potenciales y una contribución directa a la contaminación medio ambiental, y en peor termino, un aporte directo al crimen organizado del país. 


\section{Cuantificación de residuos sólidos}

La cuantificación se realizó en base a la identificación de 6 zonas (Figura 1).

\section{Figura 1. Cuantificación de residuos sólidos}

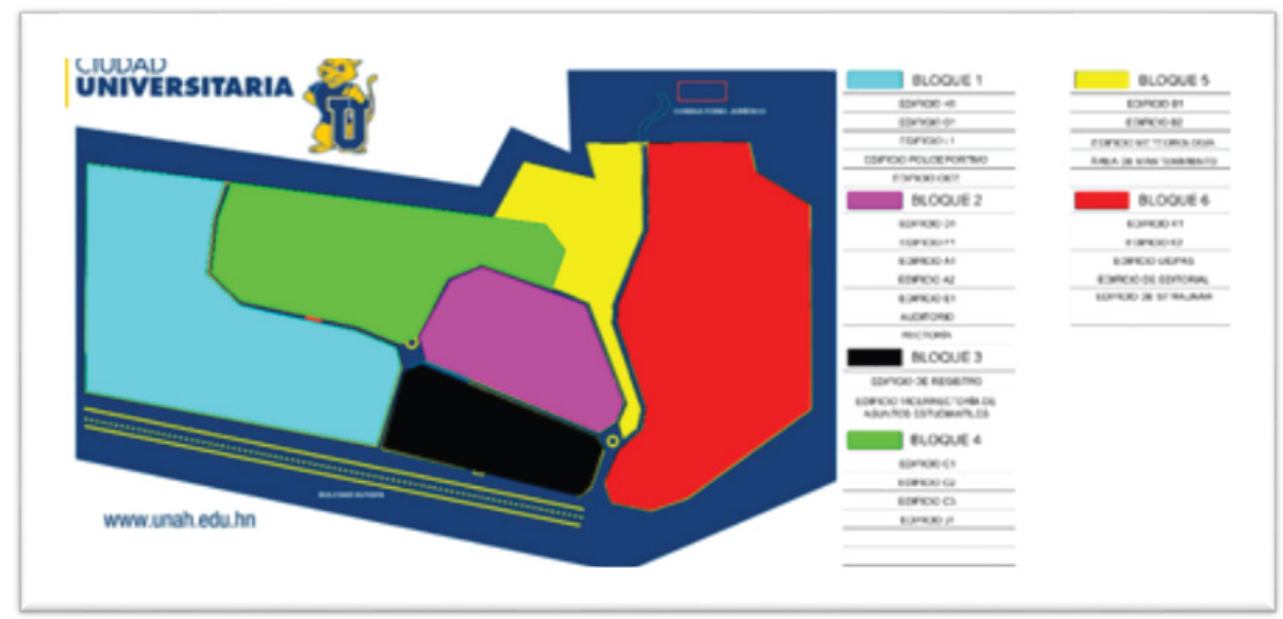

Fuente: elaboración propia

Figura 2. Distribución de contenedores de recolección de residuos en Ciudad Universitaria

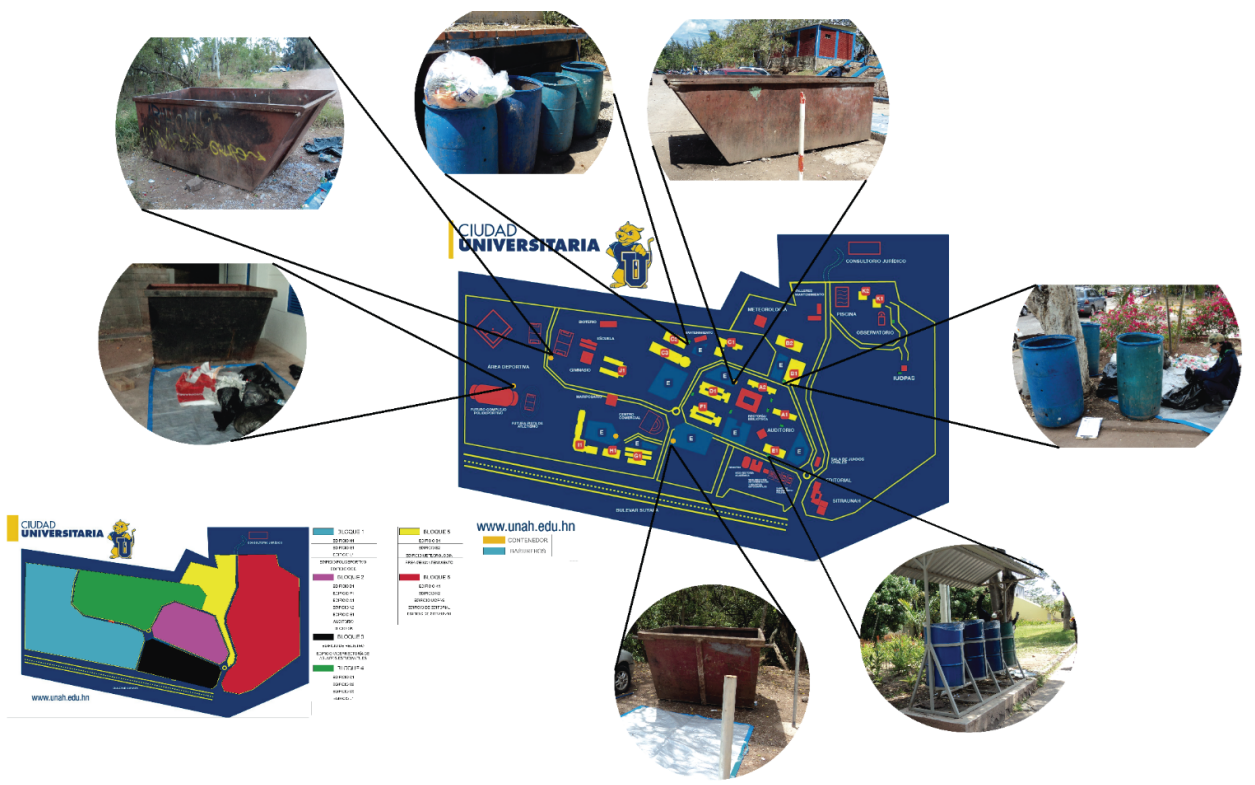

Fuente: elaboración propia 
El Campus Ciudad Universitaria está ubicado en la capital del país, fue desglosado en 6 zonas las cuales se realizaron numerosos análisis para la recolección de las muestras cuantitativas. El mismo cuenta con la ubicación de varios centros de acopio para recolectar residuos sólidos, teniendo un total de 5 contenedores de 5 a 10 toneladas de capacidad de almacenamiento y 10 unidades contenedoras elaboradas con barriles platicos y/o metálicos. (Ver figura 2)

Grafico 1. Porcentaje de producción de residuos sólidos por día. Ciudad universitaria UNAH.

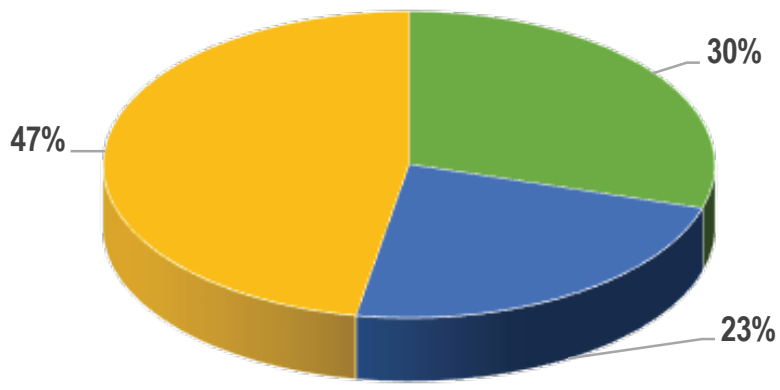

- R. Sólidos Reciclables y Reutilizables "R. Orgánicos " R. Sólidos No Reciclables ni Reutilizables

Fuente: elaboración propia

Grafico 2. Porcentaje de producción de residuos no reciclables ni reutilizables en Ciudad Universitaria.

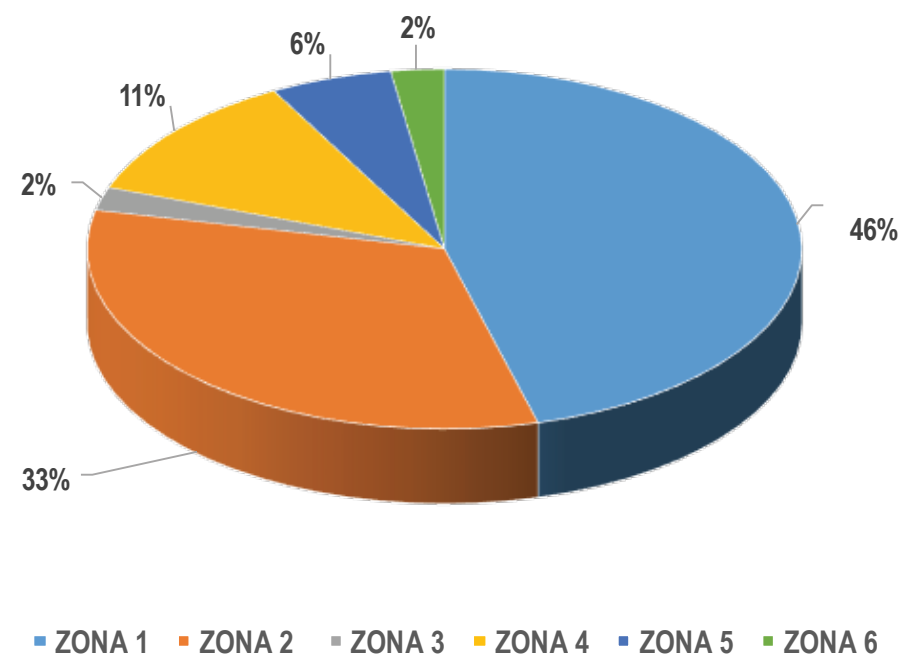

Fuente: elaboración propia 
Una vez realizada la cuantificación y tabulación de los resultados obtenidos, se obtuvo los siguientes gráficos y tablas que expresan las cantidades en libras que produce la universidad por día, semana, mes y periodo.

La Ciudad Universitaria produce en mayor porcentaje residuos sólidos no reciclables ni reutilizables, estos mismos son residuos que no pueden generar ninguna utilidad a la universidad y el mal manejo puede contribuir al deterioro del medio ambiente.Los residuos reciclables y reutilizables ocupan el $30 \%$ de la producción por periodo, y los Residuos orgánicos ocupan el último lugar en producción por periodo. De acuerdo a los datos obtenidos en la cuantificación, la zona que produce la mayor cantidad de residuos no reciclables ni reutilizables es la zona 1.

\section{Grafico 3. Residuos sólidos reciclables y reutilizables por día.}

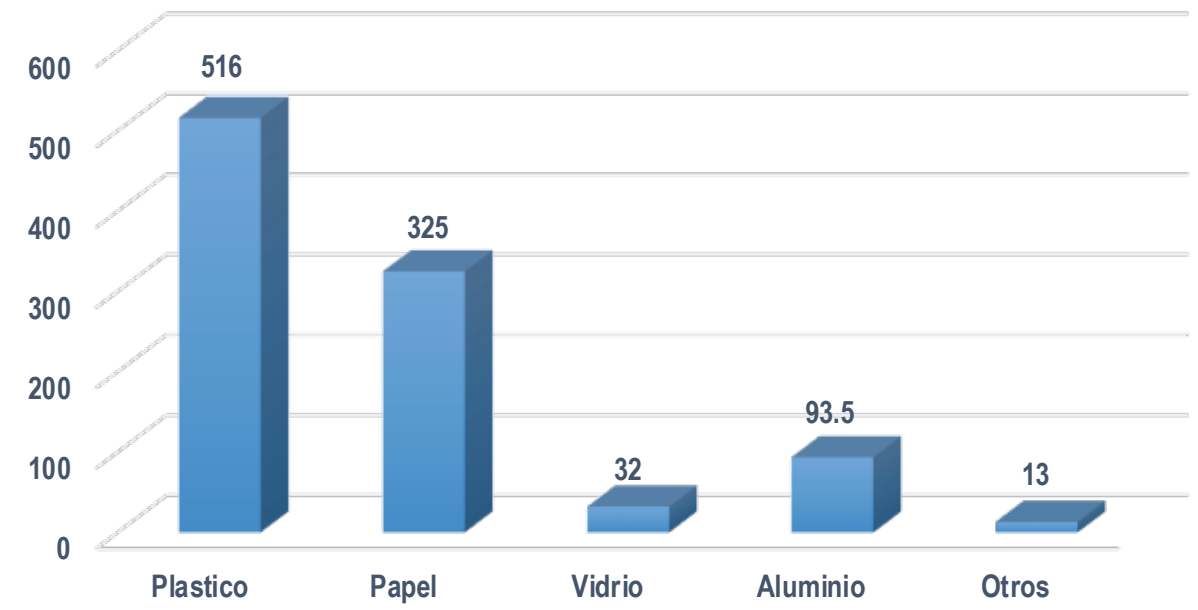

Fuente: elaboración propia

El mayor residuo que se produce a diario en Ciudad Universitaria es el plástico, seguido por el papel, se detalla en el gráfico de barras sobre la cantidad en libras de los diversos residuos sólidos. 
Gráfico 4 . Porcentaje de producción de plástico en la Ciudad Universitaria.

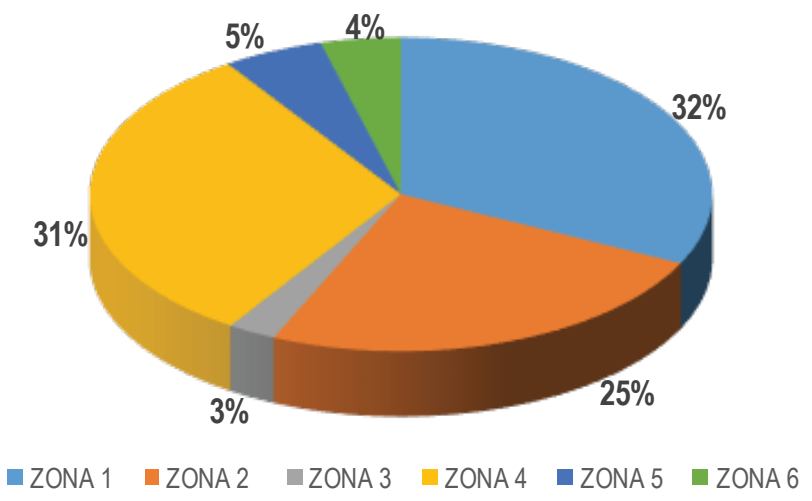

Fuente: elaboración propia

Gráfico 5 . Porcentaje de producción de papel en Ciudad Universitaria

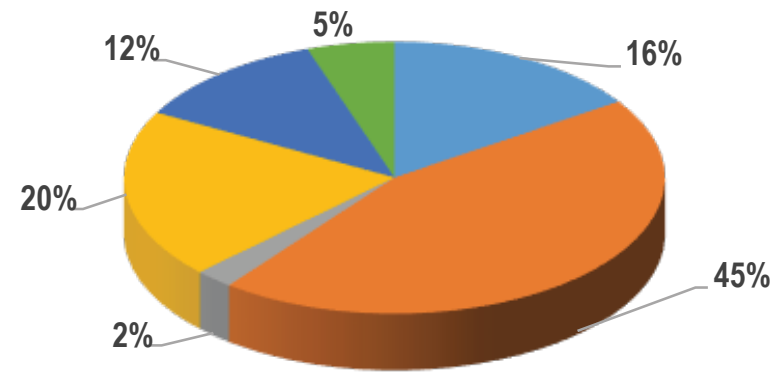

ZONA 1 ZONA $2 \square$ ZONA 3 ZONA 4 ZONA $5 \square$ ZONA 6

Fuente: elaboración propia

Grafico 6. Porcentaje de producción de aluminio en Ciudad Universitaria.

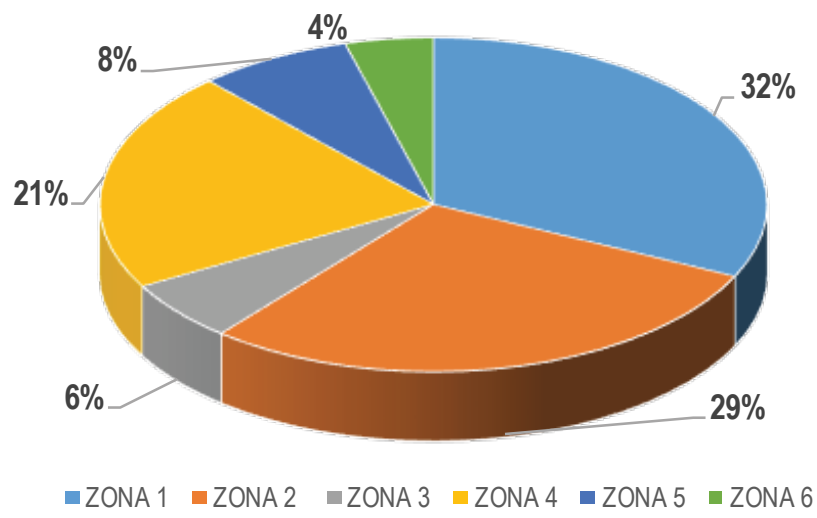

Fuente: elaboración propia 
Los resultados que obtenidos en el levantamiento cuantitativo muestran que la zona 2 es la que más produce residuos sólidos reciclables y reutilizables como ser: papel, plástico, residuos orgánicos, y otros residuos reciclables y reutilizables.

\section{Gráfico 7. Porcentaje de producción de residuos sólidos orgánicos en Ciudad Universitaria}

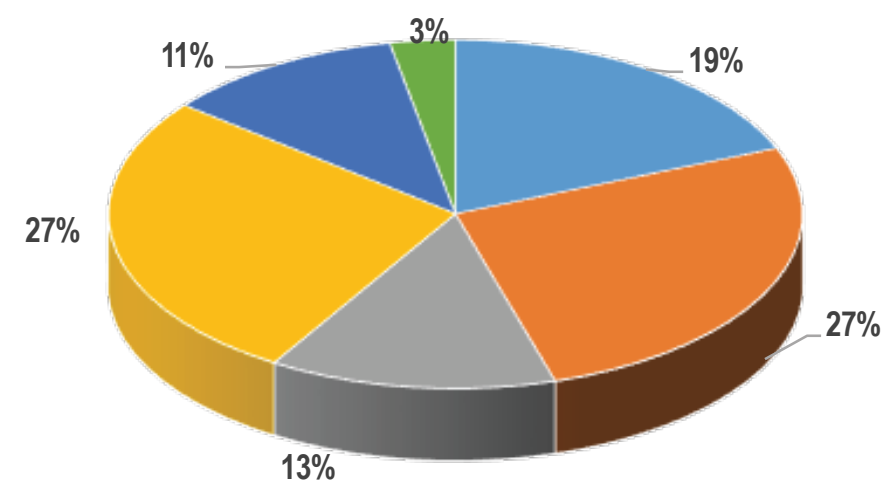

$\square$ ZONA 1 ZONA $2 \square$ ZONA $3 \quad \square Z O N A 4 \quad \square Z O N A 5 \square Z O N A 6$

Fuente: elaboración propia

La Zona 1 produce el $32 \%$ de aluminio que la Ciudad Universitaria genera por periodo, esta zona general un $27 \%$ de los residuos orgánicos.

\section{Potenciales propuestos de uso para los residuos sólidos:}

La mayoría de propuestas existentes en reciclaje y reutilización responden a soluciones artesanales y ornamentales, es decir con fines decorativos o estéticos (como adornos de botellas, floreros, maceteros, adornos con CD, entre otros), sin embargo, debido a la gran magnitud de residuos que se producen en la UNAH, es posible pensar en soluciones más operativas y de escala mayor para problemas concretos, como lo ha hecho la Alcaldía Municipal del Distrito Central, en la localidad del Museo para la Identidad Nacional MIN, en donde se construyó con botellas de plástico una cortina que cubre un gran tramo peatonal proporcionando sombra a los peatones, lo que se adecuaría a la perfección en la entrada peatonal de la UNAH y estéticamente dotaría de mucha vistosidad a la UNAH-CU.

Por otro lado, estas son algunas posibles aplicaciones de reutilizar los Residuos sólidos con un alcance mayor que se pueden señalar: 
Tabla 1. Base de datos de cuantificación de residuos

\begin{tabular}{|c|c|c|c|c|c|c|}
\hline \multicolumn{7}{|c|}{ Tabla base de datos de cuantificación de residuos } \\
\hline \multicolumn{7}{|c|}{ Total libras de residuos UNAH Ciudad Universitaria por día } \\
\hline \multicolumn{5}{|c|}{ Reciclables y reutilizables } & \multirow[t]{2}{*}{ Orgánico } & \multirow[t]{2}{*}{ No reciclables } \\
\hline Plástico & Papel & Vidrio & Aluminio & Otros & & \\
\hline 516 & 325 & 32 & 93.5 & 13 & 765.75 & $1,561.75$ \\
\hline \multicolumn{7}{|c|}{ Total libras de residuos UNAH Ciudad Universitaria por semana } \\
\hline \multicolumn{5}{|c|}{ Reciclables y reutilizables } & \multirow[t]{2}{*}{ Orgánico } & \multirow[t]{2}{*}{ No reciclables } \\
\hline Plástico & Papel & Vidrio & Aluminio & Otros & & \\
\hline 2,580 & 1,625 & 160 & 467.5 & 65 & $3,828.75$ & $7,808.75$ \\
\hline \multicolumn{7}{|c|}{ Total libras de residuos UNAH Ciudad Universitaria por mes } \\
\hline \multicolumn{5}{|c|}{ Reciclables y reutilizables } & \multirow[t]{2}{*}{ Orgánico } & \multirow[t]{2}{*}{ No reciclables } \\
\hline Plástico & Papel & Vidrio & Aluminio & Otros & & \\
\hline 10,320 & 6,500 & 640 & 1,870 & 260 & 15,315 & 31,235 \\
\hline \multicolumn{7}{|c|}{ Total libras de residuos UNAH Ciudad Universitaria por periodo } \\
\hline \multicolumn{5}{|c|}{ Reciclables y reutilizables } & \multirow[t]{2}{*}{ Orgánico } & \multirow[t]{2}{*}{ No reciclables } \\
\hline Plástico & Papel & Vidrio & Aluminio & Otros & & \\
\hline 30,960 & 19,500 & 1,920 & 5,610 & 780 & 45,945 & 93,705 \\
\hline
\end{tabular}

Fuente: elaboración propia

\section{Fabricación de gasolina y diésel}

Dentro de los posibles usos encontramos una iniciativa que va de la mano de la vanguardia en avances de reciclaje, en el campo de las ciencias, se encuentra en las Universidades de Costa Rica y en México. Recientemente han descubierto que, de los residuos plásticos, a través de un proceso de reconversión se ha logrado producir Gasolina y Diésel, con la técnica llamada ingeniería de retroceso, la cual consiste en que la materia prima que proviene del petróleo, para la elabora de un producto como plástico, se puede regresar a sus derivados del petróleo, lo cual indica que por cada $100 \mathrm{~kg}$ se puede producir 50 litros de diésel. (NOTIMEX, 2016) En el caso dela producción de plásticos de la UNAH se encontró que se producen 10,320 libras de plásticos por mes, lo que significaría una producción total de 2,345 litros de hidrocar- 
buros al mes. Esto implicaría que la UNAH, a través de sus diferentes facultades, impulse iniciativas de investigadores y alumnos que tengan las capacidades de aplicar este tipo de procesos.

\section{Utilización de PEPS en agua y vivienda}

Por otro lado, con los plásticos PEPS se podría fabricar calentadores de agua caseros, cosechadoras de agua de vivienda, ladrillos, entre otros, lo que podría significar una solución para la construcción y adecuaciones de viviendas (Ecolnventos, 2014).

\section{Material orgánico}

Con el material orgánico, se podría fabricar abono para cultivos orgánicos, gas propano, entre otros, lo que implicaría el involucramiento de diversas carreras como biología y las asignaturas como educación ambiental, en la promoción de hábitos como la permacultura y otras prácticas medioambientales sostenibles (Paecke, 2014).

Con todo el material orgánico se podría construir una fuente de insumos orgánicos para uso de la misma universidad y para su comercialización, además de ser un insumo básico para la enseñanza de prácticas orgánicas, necesarias en una sociedad como la hondureña, con altos problemas de contaminación. De este modo la UNAH entraría en la vanguardia de prácticas de auto sostenibilidad en relación al ambiente.

Análisis

Los resultados concluyen que existe un enorme potencial en los residuos sólidos que produce la UNAH, lo cual implica un insumo muy importante en futuras investigaciones y proyectos sobre reutilización. Tener claridad plena en base a datos sobre el impacto ambiental y el potencial de los recursos que la UNAH produce, constituye un avance necesario, en la promoción y consolidación de una universidad socialmente responsable con el medio ambiente, y coherente con los avances investigativos en el tema del reciclaje y la reutilización.

Para cumplir con un verdadero proceso de clasificación y reciclaje o reutilización se debe trabajar de manera trasversal con toda la comunidad universitaria, docentes, estudiantes, personal administrativo y de mantenimiento, ya que la idea de un Sistema Integral exige el involucramiento de toda la comunidad universitaria, lo cual deberá de ser prioridad y responsabilidad de las entidades correspondientes en la UNAH-CU. 
El estudio tuvo la gran limitación de ser un estudio descriptivo, por lo que su alcance se limita a describir y proponer, sin tener mayor incidencia institucional en los tomadores de decisiones para impulsar realmente un proceso de reciclaje y reutilización en la UNAH-CU.

Sobre las propuestas de uso existe la gran necesidad de involucrar a los distintos campos del conocimiento que pueden aportar a través de sus disciplinas estudios e iniciativas sobre el uso de los residuos. En el caso de la "ingeniería de reversa", proceso utilizado como método de conversión del plástico y otros derivados del petróleo, en combustibles, se debe de precisar orientar dichas propuestas a los campos correspondientes como Ingeniería Química, o en el caso de los materiales orgánicos, la carrera de Biología, podría ocuparse de crear iniciativas en base a la cantidad de residuos sólidos que se producen en la UNAH-CU

\section{DISCUSIÓN}

La investigación es de tipo explicativo descriptivo, teniendo un componente de tipo cuantitativo como también un componente cualitativo, lo que la convierte en una investigación de carácter mixto, esta investigación puede ser como el inicio o la motivación para poder ahondar más en esta área o temática y por la importancia actual del sostenimiento del medio ambiente.

Como se puede observar en las recomendaciones se expresa algunas ideas para incursionar en alguna investigación tecnológica, en otras universidades ya se están realizando investigación tecnológica utilizando la teoría de la ingeniería inversa.

\section{RECOMENDACIONES}

Propulsar la creación de un SIGRS con las condiciones simbólicas y materiales estructurales necesarias, que aseguren un proceso de clasificación de la basura para su respectiva reutilización y reciclaje. Para ello, la UNAH deberá de involucrar a la comunidad universitaria en el proceso de sensibilización sobre la responsabilidad ambiental de la UNAH, la importancia del reciclaje y la clasificación de los residuos. Realizar campañas masivas de capacitación, con el fin de inculcar la cultura de la clasificación de los residuos sólidos, como también instalar en todos los niveles de los 
edificios, recipientes de recolectores con la diferente simbolización, al igual instalar contenedores con su debida simbología de clasificación en puntos estratégicos.

La UNAH, deberá de instruir al personal encargado de los vehículos recolectores, para que sistematice los horarios de recolectar los residuos sólidos de acuerdo a su clasificación, evitando de esa manera que en el mismo vehículo se recolecte todos los desechos sólidos y evita que fuese en vano el trabajo previo de la clasificación. Además, deberá de incentivar, a través de las diferentes facultades, escuelas y carreras a realizar investigación de los posibles usos científicos de estos residuos sólidos, impulsando y creando iniciativas lúdicas, sostenibles, viables y pertinentes para la reutilización y reciclaje de los residuos, vinculadas a las necesidades de la UNAH y de la sociedad e incorporando saberes y conocimientos científicos especializados en el campo de la reutilización y el reciclaje.

\section{CONCLUSIONES}

Primero, es necesario aceptar el hecho de que la UNAH no cuenta con un Sistema Integral de Gestión de Residuos Sólidos SIGRS como tal, se produce una cantidad considerablemente de residuos sólidos reciclables y reutilizables por lo cual, la gestión de los residuos se realiza de manera pragmática y ortodoxa, es decir, se realiza la labor sin la mayor atención a un proceso normativo operacional integral y auto sostenible, propiamente adecuado a una institución de alto carácter científico, técnico y académico como la UNAH.

Segundo, no se cuenta con verdadero proceso de clasificación, ni de un ente que dé seguimiento a este proceso de manejo de residuos sólidos, es necesario reconocer el tipo de racionalidad instrumental que embiste a los residuos sólidos con una carga peyorativa de inservible, esto se encierra en el uso del término basura, y en las practicas que giran alrededor de ellas, plasmadas en los distintos discursos sobre el nivel de depósito. Esto exige a la UNAH un trabajo profundo a nivel simbólico para modificar dichas prácticas irreflexivas y construir una conciencia ambiental operativa y con un impacto real en el imaginario colectivo de la UNAH.

Tercero, la población universitaria, no tiene una cultura de clasificación de los residuos sólidos, razón por la cual es imposible realizar la recolección de los mismos en forma ordenada y expedita para poder clasificar dichos residuos sólidos. 
Por último, existe un problema visible en la infraestructura del proceso de gestión de residuos actual, sin embargo, es necesario reconocer que existen los cimientos y esfuerzos por parte de diversos sectores de la UNAH para la construcción y puesta en marcha de un Sistema Integral de Residuos, por lo cual se deben de reforzar esos esfuerzos, creando normativas, protocolos y dotando de los materiales y capacidades necesarias para el personal involucrado en dicho proceso.

\section{AGRADECIMIENTO}

Agradezco a la Universidad Nacional Autónoma de Honduras por dar la apertura de realizar este proyecto de investigación; a la Dirección de investigación Científica y Postgrados (DICYP), por la guía que nos brindaron en el desarrollo y presentación de este artículo, a la Escuela de Arquitectura de la UNAH, quien nos brindó el apoyo necesario para desarrollar este proyecto; a los Estudiantes Wilmer Zorto y Yelenia Rodríguez, gracias a ellos se dio inició este proyecto. Por último, a mis compañeros de Investigación Lic. Jorge Fernando Pacheco Dubón y Arq. Juan Carlos Montalván Flores porque en esta armonía grupal lo hemos logrado.

\section{BIBLIOGRAFÍA}

Ecolnventos. (2014, julio 21). Ecolnventos. Recuperado el 12 de mayo de 2016, de Ecolnventos:http://ecoinventos.com/6-propuestas-de-calentadores-solarescaseros/

NOTIMEX. (2016). Dinero en Imagen. Recuperado de: http://www.dineroenimagen.com/2016-01-17/67374

Paecke, L. S. (2014). Eldefinido.cl. Recuperado de: http://www.eldefinido.cl/actualidad/pais/1810/Transforma_tu_basura_en_un_recurso_natural/ 